Article

\title{
Analysis of Artifacts Caused by Pulse Imperfections in CPMG Pulse Trains in NMR Relaxation Dispersion Experiments
}

\author{
Tsuyoshi Konuma ${ }^{1}$ (D), Aritaka Nagadoi ${ }^{2}$, Jun-ichi Kurita ${ }^{1}$ and Takahisa Ikegami ${ }^{1, *(1)}$ \\ 1 Graduate School of Medical Life Science, Yokohama City University, 1-7-29 Suehiro-cho, Tsurumi-ku, \\ Yokohama 230-0045, Japan; konumax@yokohama-cu.ac.jp (T.K.); kurita-varian@yokohama-cu.ac.jp (J.K.) \\ 2 Bruker Japan K.K., Osaka Office, 1-8-29 Nishimiyahara, Yodogawa-ku, Osaka-shi, Osaka 532-0004, Japan; \\ Aritaka.Nagadoi@bruker.com \\ * Correspondence: ikegamit@yokohama-cu.ac.jp; Tel.: +81-45-508-7216
}

Received: 28 June 2018; Accepted: 26 July 2018; Published: 30 July 2018

\begin{abstract}
Nuclear magnetic resonance relaxation dispersion (rd) experiments provide kinetics and thermodynamics information of molecules undergoing conformational exchange. Rd experiments often use a Carr-Purcell-Meiboom-Gill (CPMG) pulse train equally separated by a spin-state selective inversion element (U-element). Even with measurement parameters carefully set, however, parts of ${ }^{1} \mathrm{H}-{ }^{15} \mathrm{~N}$ correlations sometimes exhibit large artifacts that may hamper the subsequent analyses. We analyzed such artifacts with a combination of NMR measurements and simulation. We found that particularly the lowest CPMG frequency $\left(v_{\mathrm{cpmg}}\right)$ can also introduce large artifacts into amide ${ }^{1} \mathrm{H}-{ }^{15} \mathrm{~N}$ and aromatic ${ }^{1} \mathrm{H}-{ }^{13} \mathrm{C}$ correlations whose ${ }^{15} \mathrm{~N} /{ }^{13} \mathrm{C}$ resonances are very close to the carrier frequencies. The simulation showed that the off-resonance effects and miscalibration of the CPMG $\pi$ pulses generate artifact maxima at resonance offsets of even and odd multiples of $v_{\text {cpmg, }}$, respectively. We demonstrate that a method once introduced into the rd experiments for molecules having residual dipolar coupling significantly reduces artifacts. In the method the ${ }^{15} \mathrm{~N} /{ }^{13} \mathrm{C} \pi$ pulse phase in the U-element is chosen between $x$ and $y$. We show that the correctly adjusted sequence is tolerant to miscalibration of the CPMG $\pi$ pulse power as large as $\pm 10 \%$ for most amide ${ }^{15} \mathrm{~N}$ and aromatic ${ }^{13} \mathrm{C}$ resonances of proteins.
\end{abstract}

Keywords: nuclear magnetic resonance (NMR); Carr-Purcell-Meiboom-Gill (CPMG) pulse train; relaxation dispersion; off-resonance effect; pulse imperfection

\section{Introduction}

Relaxation dispersion (rd) experiments of nuclear magnetic resonance (NMR) are one of the most commonly used methods to obtain valuable information about the kinetics and thermodynamics of molecules for which the conformations exchange between those in the major-populated visible state and in the minor-populated invisible state or among more conformational sub-states [1-4]. Recent developments of high-power rd experiments enable motions as fast as $4 \mu$ s to be detected [5-8]. Experiments intended for molecules with such characters provide effective transverse relaxation rates $\left(R_{2}{ }^{\text {eff }}\right)$ that depend on the field strength of the spin-lock [9] or on the repetition rate of refocusing $\pi$ pulses in Carr-Purcell-Meiboom-Gill (CPMG) pulse trains $[10,11]\{\delta-\pi-\delta\}_{n}$, where $\delta, \pi$, and $n$ represent a delay, the $\pi$ refocusing pulse between them, and the repeat count of spin echoes, respectively. The dependency plots a dispersion profile of $R_{2}{ }^{\text {eff }}$ for each observed spin. However, obtaining smooth dispersion curves with minimum artifacts in practice requires careful calibration of some measurement parameters [12,13]. 
In ${ }^{15} \mathrm{~N}$ spin rd experiments, the most frequently used method is a relaxation compensated (rc) CPMG pulse sequence, originally proposed by Loria et al. [14,15]. The pulse sequence contains two CPMG relaxation periods of equal and constant lengths without accompanied ${ }^{1} \mathrm{H}$ decoupling (Figure 1a). The two periods are separated by a sequence basically composed of $1 /\left(4^{1} J_{\mathrm{HN}}\right)-\pi^{\mathrm{H}} / \pi^{\mathrm{N}}-1 /\left(4^{1} J_{\mathrm{HN}}\right)-\pi^{\mathrm{H}}$, referred to hereafter as U-element, where $\pi^{\mathrm{H}} / \pi^{\mathrm{N}}$ represent $\pi$ pulses applied simultaneously to ${ }^{1} \mathrm{H}$ and ${ }^{15} \mathrm{~N}$ spins, respectively. The former $\left(\mathrm{CPMG}_{1}\right)$ and latter $\left(\mathrm{CPMG}_{2}\right)$ pulse trains begin by targeting ${ }^{15} \mathrm{~N}$ spin transverse coherences that are, respectively, anti-phase $2 N_{y} H_{z}$, and in-phase $N_{x}$ with respect to the coupled ${ }^{1} \mathrm{H}$ spins through the ${ }^{1} J_{\mathrm{HN}}$ coupling constant according to the CPMG pulse phases in an example shown in Figure 1a. Since the anti-phase and in-phase coherences evolve by the ${ }^{1} J_{\mathrm{HN}}$ coupling and exchange with each other during the intervals between consecutive refocusing $\pi$ pulses $(2 \delta)$, the mixture ratio between the two coherences depends on $\delta$ as far as either the $C P M G_{1}$ or $C P M G_{2}$ period alone is concerned. Anti-phase coherences tend to relax faster than the corresponding in-phase coherences, owing to additional dipolar interactions between the coupled ${ }^{1} \mathrm{H}$ spin and nearby surrounding ${ }^{1} \mathrm{H}$ spins by means of mutual spin flip-flops. As a result, even molecules having no exchange exhibit $R_{2}{ }^{\text {eff }}$ that depends on $\delta$. To overcome this problem, anti-phase coherences in $\mathrm{CPMG}_{1}$ are converted to the associated in-phase ones by the U-element placed between the two CPMG periods [14,15] (Figure 1b-g). Consequently, the total integrated periods as being experienced by the anti-phase and in-phase coherences are equalized. Namely, the differential relaxation rates of the in-phase and anti-phase coherences are averaged in the frame of average Hamiltonian theory [16], and the exchange rate $\left(R_{\mathrm{ex}}\right)$ alone becomes dependent on the repetition rate of the refocusing $\pi$ pulses. The conversion from ${ }^{15} \mathrm{~N}$ anti-phase to in-phase coherences corresponds to spin state-selective inversion of either one magnetization component of the ${ }^{15} \mathrm{~N}$ doublet, $N_{x / y} H_{\alpha}$ or $N_{x / y} H_{\beta}$, where $\alpha$ and $\beta$ stand for the spin-states of the coupled amide ${ }^{1} \mathrm{H}$ spin [17]. The U-element, therefore, can also be expressed as canceling the cross-relaxation between the $N_{x / y} H_{\alpha}$ and $N_{x / y} H_{\beta}$ coherences.

In rc heteronuclear transverse relaxation optimized spectroscopy (TROSY)-based CPMG experiments [18], a false large $R_{2}{ }^{\text {eff }}$ is often observed, particularly at low repetition rates of the CPMG refocusing $\pi$ pulses, even when the radio frequency (rf) magnetic field strength of the ${ }^{15} \mathrm{~N}$ or ${ }^{13} \mathrm{C} \pi$ pulses is accurately calibrated. Such a large $R_{2}$ eff derives from the small peaks compared to those that should be properly observed in the two-dimensional (2D) ${ }^{1} \mathrm{H}-{ }^{15} \mathrm{~N}$ or ${ }^{1} \mathrm{H}-{ }^{13} \mathrm{C}$ correlation spectra obtained at the corresponding CPMG frequency $\left(v_{\text {cpmg }}=1 /(4 \delta)\right)$. The lowest repetition rate is achieved by placing a single spin-echo in each side of the U-element in the rc CPMG pulse train. It is well known that a CPMG pulse train composed of even-numbered spin-echoes with the same $\pi$ pulse phase has a property of canceling some artifacts caused by pulse imperfections [19]. However, as one is an odd number, artifacts are not compensated within each CPMG period alone. Furthermore, rd profiles carrying large artifacts only at CPMG frequencies corresponding to odd numbers of spin-echoes may look similar to the profiles of spin systems exchanging slowly on the NMR chemical shift time scale. Such slow exchange exhibits damped oscillation patterns in the low $v_{\text {cpmg }}$ range of the rd curves that can be approximated by sinc functions [20]. Consequently, such artifacts may lead to incorrect information regarding the dynamics of the molecules being observed. 
(a)

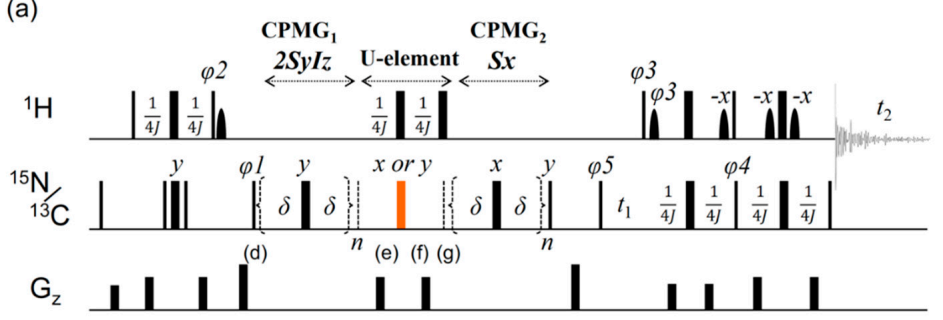

(b)

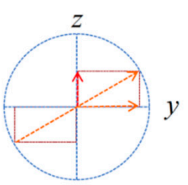

(d)

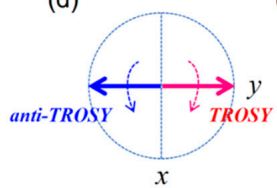

(e)

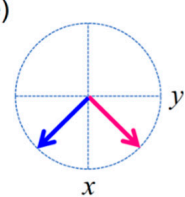

(c)

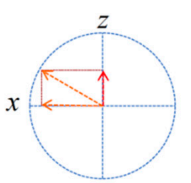

(f)

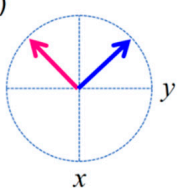

(g)

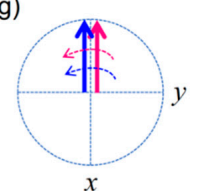

Figure 1. (a) A scheme of the transverse relaxation optimized spectroscopy (TROSY)-based rc Carr-Purcell-Meiboom-Gill (CPMG) rd pulse sequence used in the experiments for amide ${ }^{1} \mathrm{H} /{ }^{15} \mathrm{~N}$ and aromatic ${ }^{1} \mathrm{H} /{ }^{13} \mathrm{C}$ spin systems. Narrow and wide bars depict $90^{\circ}$ and $180^{\circ}$ pulses, respectively. All pulses were applied with phase $x$, unless indicated otherwise. The ${ }^{1} \mathrm{H}$ pulses were applied at the water resonance with an $\mathrm{rf}$ field strength of $25 \mathrm{kHz}(10.0 \mu$ s as a $\pi / 2$ pulse width), except for bell-shaped pulses, which were applied as a $1 \mathrm{~ms}$ water-selective $\pi / 2$ sinc-shaped pulse. All ${ }^{15} \mathrm{~N}$ and ${ }^{13} \mathrm{C}$ pulses were applied at 119 and $125 \mathrm{ppm}$ with rf field strengths of 6250 and 12,500 Hz (40 and $20 \mu \mathrm{s}$ as $\pi / 2$ pulse widths), respectively, at a ${ }^{1} \mathrm{H}$ static magnetic field strength of $800 \mathrm{MHz}$. The constant-time CPMG period is divided into two parts (20 ms for each). For each $C P M G_{1}$ and $C P M G_{2}$ period, a spin-echo, $\{\delta-\pi-\delta\}$, was repeated $n$ times. $J$ is the scalar coupling constant between the ${ }^{1} \mathrm{H}(I)$ spin and the amide ${ }^{15} \mathrm{~N}$ or aromatic ${ }^{13} \mathrm{C}(S)$ spin. Pulse phases (Bruker) were cycled as follows: $\phi_{1}=\{4(x), 4(-x)\}, \phi_{2}=\{8(y)$, $8(-y)\}, \phi_{3}=\{-y\}, \phi_{4}=\{-y\}, \phi_{5}=\{y,-x,-y, x\}$, and $\phi_{\text {rec }}=\{y,-x,-y, x, 2(-y, x, y,-x), y,-x,-y$, $x$. The ${ }^{15} \mathrm{~N}$ or ${ }^{13} \mathrm{C}$ indirect dimension was obtained by recording a second data set with, $\phi_{3} \rightarrow \phi_{3}$ $+\pi, \phi_{4} \rightarrow \phi_{4}+\pi$, and $\phi_{5}=\{y, x,-y,-x\}$. For each $t_{1}$ increment, $\phi_{5}$ and $\phi_{\text {rec }}$ were incremented by $\pi$ radians $\left(\phi_{5} \rightarrow \phi_{5}+\pi\right.$ and $\left.\phi_{\text {rec }} \rightarrow \phi_{\text {rec }}+\pi\right)$ to shift the axial peaks to the edges of the spectrum; (b) The effective magnetic field made by the $S$ spin offset from the carrier frequency, represented by an arrow along the $z$-axis, and the rf field frequency of the CPMG $\pi$ pulses along the $y$-axis in the $\mathrm{CPMG}_{1}$ period, viewed from the $+x$ direction; (c) The effective magnetic field in the $\mathrm{CPMG}_{2}$ period, viewed from the $+y$ direction; (d) The anti-phase magnetization $-2 S_{y} I_{z}$ at the beginning of the $\mathrm{CPMG}_{1}$ period, viewed from the $+z$ direction. The TROSY $\left(+S_{y} I_{\beta}\right)$ and anti-TROSY $\left(-S_{y} I_{\alpha}\right)$ magnetization components are represented by red and blue arrows, respectively. For simplicity, any chemical shift evolution is ignored throughout the $\mathrm{CPMG}_{1}, \mathrm{U}$-element, and $\mathrm{CPMG}_{2}$ periods. The curved and dashed arrows indicate the rotational direction of the magnetization vectors about the effective magnetic field, when it is assumed that the delay $\delta$ is negligibly short. The situation is rather similar to that in $S$ spin lock in $T_{1 \rho}$ experiments instead of CPMG spin echoes; (e) The two magnetizations having evolved by ${ }^{1} J$ coupling for the $1 /\left(4^{1} J\right)$ period just before application of the spin $S \pi$ pulse in the U-element; (f) The magnetization vectors just after the spin $I \pi$ pulse and the spin $S \pi$ pulse of phase $y$. Both magnetizations will evolve and approach the $-x$-axis; $(g)$ The in-phase magnetization $-S_{x}$ at the start of the $C P M G_{2}$ period. The TROSY and anti-TROSY magnetization components are expressed as $-S_{x} I_{\beta}$ (red) and $-S_{x} I_{\alpha}$ (blue), respectively. When the rotational directions of the magnetizations in the two CPMG periods are compared, it turns out that the anti-TROSY magnetization is rotated about the effective magnetic field in the same sense, while the TROSY magnetization is rotated in the opposite sense. 
Vallurupalli et al. [21] presented a method for accurately measuring the amide ${ }^{15} \mathrm{~N}$ spin $\mathrm{rd}$ of the respective TROSY and anti-TROSY magnetization components that are split along the ${ }^{15} \mathrm{~N}$ dimension by the ${ }^{1} J_{\mathrm{HN}}$ scalar coupling and the ${ }^{1} D_{\mathrm{HN}}$ residual dipolar coupling (RDC) for each amide resonance of protein molecules aligned along a static magnetic field in an alignment medium. To estimate RDC values for transiently populated conformations in minor states, they proposed a modified rc CPMG pulse sequence, which contained another pulse sequence, referred to as P-element hereafter, instead of the U-element. The P-element is different from the U-element in that the former has ${ }^{15} \mathrm{~N}$ $\pi / 2$ pulses at both ends $\left((\pi / 2)^{\mathrm{N}}-1 /\left(4^{1} J_{\mathrm{HN}}\right)-\pi^{\mathrm{H}} / \pi^{\mathrm{N}}-1 /\left(4^{1} J_{\mathrm{HN}}\right)-(\pi / 2)^{\mathrm{N}} / \pi^{\mathrm{H}}\right)$. The first $\pi / 2$ pulse is applied along $\pm y$ axes at the beginning of the P-element and the other one is applied along $\pm x$ axes at the end of the period without the receiver phase inverted. These $\pi / 2$ pulses are necessary to remove unwanted magnetization generated by evolution by the sum of the scalar coupling $\left({ }^{1} J_{\mathrm{HN}}\right)$ and non-uniform residual dipolar coupling $\left({ }^{1} D_{\mathrm{HN}}\right)$ constants for samples aligned along the static magnetic field. As described in detail later, the difference is not strongly related to artifact suppression, as far as the observed systems are isotropic with no RDC. In the specific rc CPMG pulse sequence, they also introduced a smart way of minimizing artifacts. By adjusting the phase of the ${ }^{15} \mathrm{~N} \pi$ pulse in the P-element, artifacts accumulated in the $\mathrm{CPMG}_{1}$ period can be compensated for in the $\mathrm{CPMG}_{2}$ period for either the TROSY or anti-TROSY magnetization component. Thus, artifacts are canceled, even when an odd number of spin-echoes are placed in each CPMG period, as if the two CPMG pulse trains were tightly arranged for the total number of spin-echoes to be even.

With knowledge of the theory, we rather focused on the detailed analysis of how artifacts are generated in rc CPMG experiments. The combination of our NMR experiments and simulation has clarified the reasons for the following phenomena. Artifacts are often observed at the lowest $v_{\text {cpmg }}$ $(n=1)$. They appear even in peaks resonating near the $S$ spin carrier frequency. They do not linearly increase with the offset frequency, unlike general intuition. They can often disappear by just shifting the carrier frequency by as small as tens of hertz. Peak intensities in rc CPMG experiments are vulnerable to artifacts unlike those in the $R_{2}$ relaxation measurement experiments in popular $R_{1}, R_{2}$, $\left\{{ }^{1} \mathrm{H}\right\}-{ }^{15} \mathrm{~N}$-nuclear Overhauser effect (NOE) analyses [22]. We here demonstrate that the method once introduced into the rd experiments for molecules having RDC [21,23] can also be applied to molecules with normal isotropic tumbling in order to significantly reduce unexpectedly large artifacts on $R_{2}{ }^{\text {eff }}$, which may hamper further analyses. The correct U-element also tolerates miscalibration of the rf magnetic field strength of the CPMG $\pi$ pulses with a deviation as large as $\pm 10 \%$. The method is effective for suppressing such artifacts against the pulse miscalibration and off-resonance effects not only in ${ }^{15} \mathrm{~N}$ TROSY rd CPMG experiments but also for the corresponding aromatic ${ }^{13} \mathrm{C}$ experiments. Our simulation showed that the off-resonance effect and pulse miscalibration generated artifacts at distinct off-resonance positions. This method is immediately and easily implementable in a wide range of rc CPMG sequences, but it must be noted that the correct phase of the central $\pi$ pulse in the U- or P-element is different depending on the spectrometer designs. The above-mentioned mechanisms are illustrated by a vector model, which may explain them more easily and intuitively than the average Hamiltonian-based calculation.

\section{Results}

\subsection{Artifacts Observed in Amide ${ }^{15} \mathrm{~N}$ Spin $r$ d Experiments}

To see the influence of possible miscalibration and off-resonance effects of CPMG refocusing $\pi$ pulses on the measured rd profiles, we used a small and stable protein ChiA1, for which no significant supra-millisecond conformational exchange was found in a previous ${ }^{15} \mathrm{~N}$ spin relaxation analysis [24]. Figure 2a shows the rd profiles of Lys-673 as a typical example, obtained by a TROSY-based $\mathrm{rc}^{15} \mathrm{~N}$ spin CPMG pulse sequence without ${ }^{1} \mathrm{H}$ continuous-wave decoupling during a pair of CPMG periods [21]. When the ${ }^{15} \mathrm{~N} \pi$ pulse in the middle of the U-element was applied with phase $y$, almost all the amide resonances exhibited flat rd profiles, as shown with the red profile in Figure 2a. This result is consistent 
with the observation that ChiA1 underwent no supra-millisecond significant conformational exchange. However, when the ${ }^{15} \mathrm{~N} \pi$ pulse of the U-element was applied along the axis shifted by $90^{\circ}$ (i.e., $x$ ), large artificial $R_{2}{ }^{\text {eff }}$ values were found as shown in the blue profile in Figure 2a, particularly at the lowest $v_{\text {cpmg }}$ value $(25 \mathrm{~Hz})$, where a single ${ }^{15} \mathrm{~N}$ spin-echo was placed within each CPMG period ( $\delta=10 \mathrm{~ms}$, the total relaxation time, $4^{*} \delta,=40 \mathrm{~ms}$ ). The ${ }^{1} \mathrm{H} /{ }^{15} \mathrm{~N} 2 \mathrm{D}$ planes corresponding to $v_{\mathrm{cpmg}}$ of $25 \mathrm{~Hz}$ are shown in Figure 2b,c. The peak of Lys-673 in the spectrum that was obtained with the ${ }^{15} \mathrm{~N} \pi$ pulse phase in the U-element set to $x$ was smaller in height by $12.3 \%$ than that obtained with phase $y$. As later described in detail, this happened because the U-element containing the $\pi$ pulse with phase $x$ selectively inverted the anti-TROSY magnetization component, instead of the TROSY counterpart. Consequently, the pulse sequence accumulated imperfections related to the CPMG $\pi$ pulses, including the off-resonance effects and power miscalibration, on the TROSY magnetization component without canceling imperfections during the paired CPMG periods. Even such a small reduction in the peak height resulted in an apparent $3.3 \mathrm{~Hz}(=-\ln (1-0.123) / 0.04)$ increase in $R_{2}{ }^{\text {eff }}$, which leads to the failure of further analysis or to the derivation of incorrect parameters.

Similar artifacts were also seen in peaks that resonated at frequencies very close to the carrier frequency (Figure 2d). Shifting the ${ }^{15} \mathrm{~N}$ carrier frequency by as small as $1.0 \mathrm{ppm}(81.1 \mathrm{~Hz})$ dramatically changed the appearance of artifacts. These observations suggested that the correlation between the magnitudes of artifacts and the ${ }^{15} \mathrm{~N}$ frequency offsets cannot be expressed with simple linearity. To check whether such artifacts were caused by any systematic errors related to machine or temperature instability during the long measurement time $(33 \mathrm{~h})$, we ran the pulse program in an interleaved manner. Practically, the repeat count $n$ of the CPMG spin-echoes was randomly selected from a list before scans were accumulated, but no improvement was obtained in the results. 
(a)

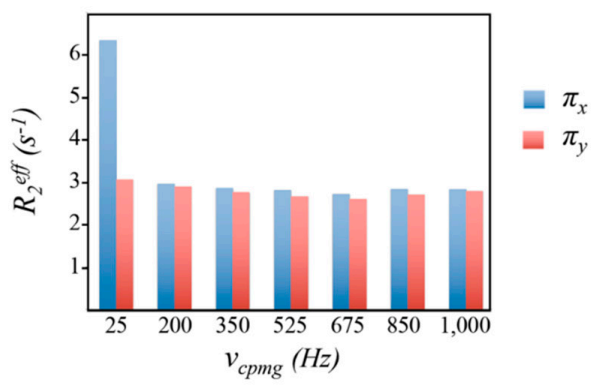

(b)

(c)

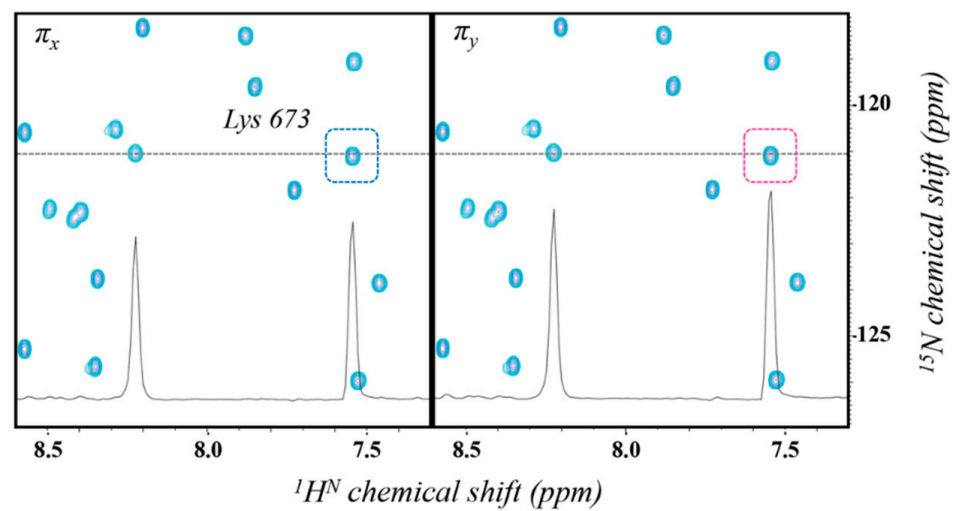

(d)

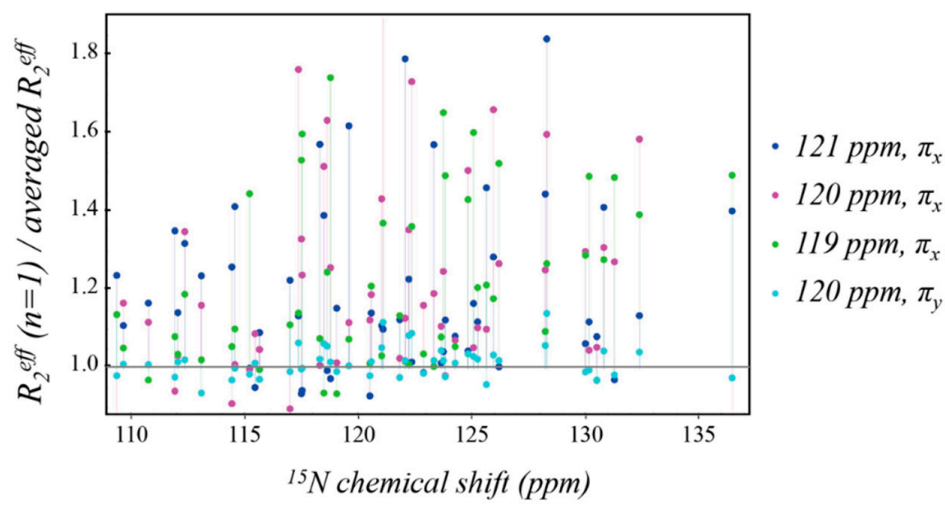

Figure 2. (a) Comparison of rd profiles of Lys-673 of $\left[{ }^{15} \mathrm{~N}\right]-\mathrm{ChiA} 1$. The data were obtained with the ${ }^{15} \mathrm{~N} \pi$ pulse in the middle of the U-element applied along the $x$-axis (blue) and $y$-axis (red) in a TROSY-based rc ${ }^{15} \mathrm{~N}$ spin CPMG rd experiment. The vertical axis stands for the effective transverse relaxation rates $\left(R_{2}{ }^{\text {eff }}\right)$, estimated from the intensity ratio of each amide ${ }^{1} \mathrm{H} /{ }^{15} \mathrm{~N}$ correlation peak obtained in the presence of the CPMG pulse trains $(40 \mathrm{~ms}$ in total) to the corresponding one obtained in their absence. The horizontal axis stands for the CPMG frequency, $v_{\text {cpmg }}=1 /(4 \delta)$; $(\mathbf{b}, \mathbf{c})$ Two-dimensional ${ }^{1} \mathrm{H} /{ }^{15} \mathrm{~N}$ TROSY-HSQC planes measured with the ${ }^{15} \mathrm{~N} \pi$ pulse in the U-element applied along the $x$-axis $(\mathbf{b})$ and $y$-axis (c), respectively, at the lowest $v_{\text {cpmg value }}(25 \mathrm{~Hz}$, a spin-echo repetition count $n$ of 1$)$. The amide peaks of Lys-673, exhibiting the abnormal value as shown in (a), are boxed. One-dimensional cross-sections along the ${ }^{1} \mathrm{H}^{\mathrm{N}}$ dimension at the ${ }^{15} \mathrm{~N}$ frequency indicated by the dashed lines are shown in the 2D spectra; (d) Artifacts observed in the spectra obtained with different ${ }^{15} \mathrm{~N}$ carrier frequencies. The horizontal axis represents the amide ${ }^{15} \mathrm{~N}$ chemical shifts of ChiA1. The vertical axis represents the ratio of $R_{2}{ }^{\text {eff }}$ obtained at $n=1$ ( $v_{\text {cpmg }}$ of $25 \mathrm{~Hz}$ ) to the average of the remaining six $R_{2}{ }^{\text {eff }}$ values obtained at $n=8$ (200), 14 (350), 21 (525), $27(675), 34(850)$, and $40(1000 \mathrm{~Hz})$ for each ${ }^{1} \mathrm{H} /{ }^{15} \mathrm{~N}$ correlation. Blue, magenta, and green points show the data obtained with the ${ }^{15} \mathrm{~N}$ carrier frequency placed at 121, 120, and $119 \mathrm{ppm}$, respectively, and with the central $\pi$ pulse in the U-element applied along the $x$-axis. Cyan points indicate the data with the $\pi$ pulse applied along the $y$-axis and the carrier frequency set at $120 \mathrm{ppm}$. The experiments were performed using a Bruker spectrometer $(800 \mathrm{MHz})$. 
While the CPMG $\pi$ pulses are applied, magnetizations describing longitudinal and transverse trajectories relax at different rates $R_{1}$ and $R_{2}$, respectively. The differential relaxation rates may cause the $R_{2}{ }^{\text {eff }}$ values to depend on the number of $\pi$ pulses applied during a constant period, i.e., $v_{\text {cpmg. }}$ A few groups have demonstrated that the application of four consecutive CPMG refocusing $\pi$ pulses with phases $\{y, y, x,-x\}$ for $\mathrm{CPMG}_{1}$ and $\{x, x, y,-y\}$ for $\mathrm{CPMG}_{2}$, respectively, suppresses many artifacts by mixing the transverse $\left(R_{2}\right)$ and longitudinal $\left(R_{1}\right)$ relaxation rates of ${ }^{15} \mathrm{~N}$ magnetization during the pulse application periods [25-28]. The method, however, did not improve the result for ChiA1. This may occur because the molecular weight of ChiA1 (5039 Da) is not large enough for the differential $R_{1}$ and $R_{2}$ relaxation rates to generate significant artifacts. In the first place, large artifacts were most often observed at the lowest $v_{\text {cpmg }}$ value, where each CPMG period contains only one spin-echo, and the above-mentioned phase combinations cannot be incorporated. We also supplemented ${ }^{15} \mathrm{~N} \pi$ pulses for the duty cycle compensation in the relaxation delay ( $3.5 \mathrm{~s}$ ) before the pulse sequence, so that the total number of ${ }^{15} \mathrm{~N}$ pulses to be applied, and hence the heat generated by pulses, was kept constant during one scan, but no improvement was found.

\subsection{TROSY-Based Aromatic ${ }^{13} \mathrm{C}$ Spin rd Experiments}

We also performed rc CPMG experiments to examine aromatic ${ }^{13} \mathrm{C}$ spins of ChiA1. In addition to correlations of amide ${ }^{1} \mathrm{H} /{ }^{15} \mathrm{~N}$ spins of proteins, those of aromatic ${ }^{1} \mathrm{H} /{ }^{13} \mathrm{C}$ spins also exhibit significant TROSY effects, so that the phase of the central ${ }^{13} \mathrm{C} \pi$ pulse in the U-element should be associated with the suppression of artifacts that appear in the ${ }^{13} \mathrm{C}$ TROSY resonances. Although aromatic ${ }^{1} \mathrm{H}$ spins do not exhibit as large a TROSY effect as aromatic ${ }^{13} \mathrm{C}$ spins due to smaller ${ }^{1} \mathrm{H}$ chemical shift anisotropy [29], we adjusted the phases in the TROSY-based $\mathrm{rc}^{13} \mathrm{C}$ spin CPMG pulse program to select the magnetization component resonating in the higher magnetic field in the ${ }^{1} \mathrm{H}$ dimension and in the lower magnetic field in the ${ }^{13} \mathrm{C}$ dimension among quadruplet peaks. These peaks are separated along both dimensions by the ${ }^{1} \mathrm{~J}_{\mathrm{CH}}$ scalar coupling constant between the bound ${ }^{13} \mathrm{C}$ and ${ }^{1} \mathrm{H}$ atoms in ${ }^{1} \mathrm{H}-{ }^{13} \mathrm{C}$ HSQC spectra without any decoupling pulses [30]. To avoid distortion of ${ }^{13} \mathrm{C}$ rd curves due to a large evolution by the ${ }^{1} J_{\mathrm{CC}}$ coupling during the $40 \mathrm{~ms}{ }^{13} \mathrm{C} C P M G$ period, we prepared ChiA1 labeled with ${ }^{15} \mathrm{~N}$ uniformly and ${ }^{13} \mathrm{C}$ alternately by inducing the expression in an $\mathrm{M} 9$ medium containing ${ }^{15} \mathrm{NH}_{4} \mathrm{Cl}$ and $\left[2-{ }^{13} \mathrm{C}\right]$-glucose. The correlation signals were detected from ${ }^{1} \mathrm{H}^{\varepsilon} /{ }^{13} \mathrm{C}^{\varepsilon}$ spins of Tyr and Phe [31-34].

When the ${ }^{13} \mathrm{C} \pi$ pulse in the U-element was applied with phase $x$ and the CPMG $\pi$ pulse strength was changed by $\pm 10 \%$ from that precisely calibrated with the sample, peak intensities at $v_{\text {cpmg }}$ of $25 \mathrm{~Hz}(n=1)$ decreased in the same way as in the case with amide ${ }^{15} \mathrm{~N}$ spins. These results are shown in the 1D spectra in Figure 3a. In contrast, when the phase of the ${ }^{13} \mathrm{C} \pi$ pulse in the U-element was shifted to $y$, these artifacts were minimized. The dependence of the intensities of the correlation peaks on the $\pi$ pulse phase in the U-element can also be clearly seen in the ${ }^{1} \mathrm{H} /{ }^{13} \mathrm{C} 2 \mathrm{D}$ spectra obtained at $v_{\text {cpmg }}$ of $25 \mathrm{~Hz}$ (Figure $3 \mathrm{~b}-\mathrm{d}$ ). Even if the CPMG $\pi$ pulses were applied with an rf field strength that was $10 \%$ higher than the calibrated value, the U-element with the central $\pi$ pulse of phase $y$ minimized the effect of the pulse miscalibration on reduction in the peak intensity compared to that of phase $x$. Interestingly, we found that all the phases and their cycles in the pulse sequence for selecting the aromatic ${ }^{1} \mathrm{H} /{ }^{13} \mathrm{C}$ TROSY magnetization components were exactly the same as in the case with amide ${ }^{1} \mathrm{H} /{ }^{15} \mathrm{~N}$ spin systems, including the $\pi$ pulse phase in the U-element. This occurred in at least two of our Bruker spectrometers with ${ }^{1} \mathrm{H}$ static magnetic fields of 500 and $800 \mathrm{MHz}$. In addition, the phase of the ${ }^{1} \mathrm{H} \pi / 2$ pulse at the end of the first INEPT was also found to be the same for the two cases, after adjusting it for the addition of the Boltzmann steady-state ${ }^{13} \mathrm{C} /{ }^{15} \mathrm{~N}$ magnetization. Although our experiments did not include the steady-state ${ }^{13} \mathrm{C} /{ }^{15} \mathrm{~N}$ magnetization, this would increase the observed signal intensity. 
(a)
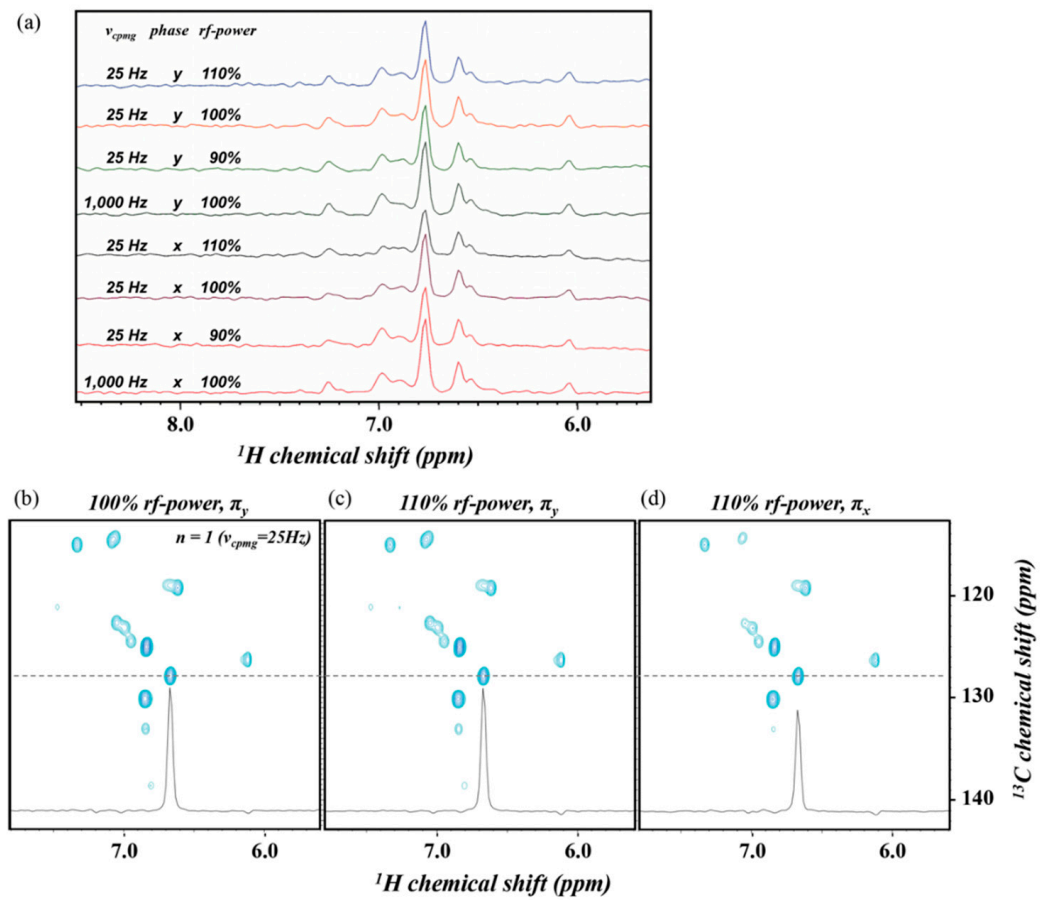

Figure 3. (a) Comparison of the peak intensities in $1 \mathrm{D}$ spectra of aromatic ${ }^{13} \mathrm{C}$ rc CPMG experiments, obtained by setting the indirect ${ }^{13} \mathrm{C}$ chemical shift evolution period $t_{1}$ to zero at $298 \mathrm{~K}$ with a ${ }^{1} \mathrm{H}$ static magnetic field of $500 \mathrm{MHz}$. The $v_{\mathrm{cpmg}}$ value $(25$ or $1000 \mathrm{~Hz})$ is indicated to the left of each 1D spectrum, together with the phase of the ${ }^{13} \mathrm{C} \pi$ pulse applied in the U-element $(x$ or $y)$, and the relative rf field strength of the ${ }^{13} \mathrm{C}$ CPMG refocusing $\pi$ pulses normalized against a calibrated pulse $(90 \%$, $100 \%$, or $110 \%$ ). The CPMG frequencies of 25 and $1000 \mathrm{~Hz}$ correspond to each of the $20 \mathrm{~ms}$ CPMG periods containing one and 40 spin-echo cycles, respectively. The rf field strength was changed by $\pm 10 \%$ ( $\pm 0.92 \mathrm{~dB})$ with respect to the calibrated power $(10 \mathrm{kHz})$. The ${ }^{13} \mathrm{C} \pi / 2$ and $\pi$ pulse lengths were maintained at 25 and $50 \mu \mathrm{s}$, respectively; (b-d) Two-dimensional aromatic ${ }^{1} \mathrm{H} /{ }^{13} \mathrm{C}$ TROSY planes

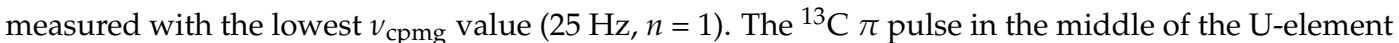
was applied with a phase of $y(\mathbf{b}), y(\mathbf{c})$, and $x(\mathbf{d})$. The rf field strength of the $\pi$ pulses in the CPMG and U-element periods was set at the exact (b) and $10 \%$ higher $(\mathbf{c}, \mathbf{d})$ power. One-dimensional cross-sections along the ${ }^{1} \mathrm{H}$ dimension at the ${ }^{13} \mathrm{C}$ frequency indicated by the dashed lines are shown in the 2D spectra. The experiments were performed using a Bruker spectrometer.

\subsection{Simulation of the rc CPMG Pulse Sequence}

We simulated the rc CPMG part of the rd experiment in an IS two-spin system, where we assumed that $I$ and $S$ spins be coupled with each other through a $100 \mathrm{~Hz}^{1} J_{\text {IS }}$ scalar coupling. No $R_{1}$ or $R_{2}$ relaxation was included in the simulation. At first, a pulse power exact on resonance was used for the $S$ spin ( $80 \mu$ s for each $\pi$ pulse), and the off-resonance effect alone was taken into account. The initial coherence $2 S_{y} I_{z}$ was made to evolve during the $\mathrm{CPMG}_{1}(20 \mathrm{~ms})$, U-element $\left(0.5 /{ }^{1} J_{\mathrm{IS}}\right)$, and $\mathrm{CPMG}_{2}(20 \mathrm{~ms})$ periods, and the normalized intensity of the $S$ spin TROSY magnetization component $S_{x} I_{\beta}$ was calculated. When the $S$ spin $\pi$ pulse in the center of the U-element was applied with phase $y$, the simulation showed peak intensities oscillating against the $S$ spin frequency offset with approximately a $10 \%$ intensity decrease at $\pm 1500 \mathrm{~Hz}$ from the carrier frequency (Figure $4 \mathrm{a}$ ). Importantly, the oscillation patterns obtained with different spin-echo repetition numbers $(n=1,2$, and 40 ) were synchronized. As far as the $S$ spin resonating at a certain frequency was concerned, the magnitudes of $S_{x} I_{\beta}$ were almost independent of $v_{\mathrm{cpmg}}$. The resulting rd profile was nearly flat with minimized artifacts, as is shown at a frequency offset of $1100 \mathrm{~Hz}$ (the magenta plot in Figure $4 \mathrm{~g}$ ). However, when the $\pi$ pulse phase in the U-element was shifted by $90^{\circ}$, the oscillation amplitude along the offset increased (Figure $4 \mathrm{~b}$ ). The rd profile at a $1100 \mathrm{~Hz}$ offset showed large artifacts, particularly 
when odd numbers of CPMG spin-echoes were applied during each CPMG period (the green plot in Figure $4 \mathrm{~g}$ ). Such a large peak intensity oscillation occurred along the frequency offset only owing to off-resonance effects of the $S$ spin. This occurs even under a condition where the pulse powers are exactly calibrated and no significant difference between $R_{1}$ and $R_{2}$ exists for the $S$ spin.

Next, we assumed in the simulation that all the $S$ spin $\pi$ pulses implemented during the two CPMG and U-element periods were miscalibrated. Practically, the rf field strength for $S$ spin pulses was reduced by $10 \%$ with the off-resonance effects occurring during implementation of the $80 \mu \mathrm{s} \pi$ pulses. We found that the application of the $\pi$ pulse in the U-element with phase $y$ also compensated for most artifacts in the TROSY magnetization component (Figure 4c). Although residual artifacts became larger, even at an offset close to the carrier frequency, the final amplitudes of the $S_{x} I_{\beta}$ component calculated with different $n$ values oscillated synchronously along the offset, being independent of $v_{\text {cpmg }}$ within an offset range of at least $\pm 1500 \mathrm{~Hz}$. The corresponding rd curves are smooth even at an offset of $1100 \mathrm{~Hz}$ (the magenta plot in Figure 4e). On the other hand, when the $\pi$ pulse in the U-element was applied with phase $x$, the final amplitude of the $S_{x} I_{\beta}$ component was reduced depending on the CPMG repetition number, $n$ (Figure $4 \mathrm{~d})$. Interestingly, a large artifact appeared at $n=1\left(v_{\text {cpmg }}=25 \mathrm{~Hz}\right)$ at an offset as small as $25 \mathrm{~Hz}$ (Figure 4f), but it almost disappeared at an offset of $50 \mathrm{~Hz}$ (Figure 4h). This simulation result was similar to the actual observation in our ${ }^{15} \mathrm{~N} \mathrm{rc} \mathrm{CPMG} \mathrm{TROSY} \mathrm{experiments,}$ namely that artifacts seemingly appeared only in randomly selected correlation peaks (Figure 2d). We also confirmed that when the anti-TROSY magnetization component $S_{x} I_{\alpha}$ was selected in the simulation, the effect described above reversed with respect to the $\pi$ pulse phase in the U-element. Namely, artifacts were more suppressed when the $\pi$ pulse was applied along the $x$-axis.

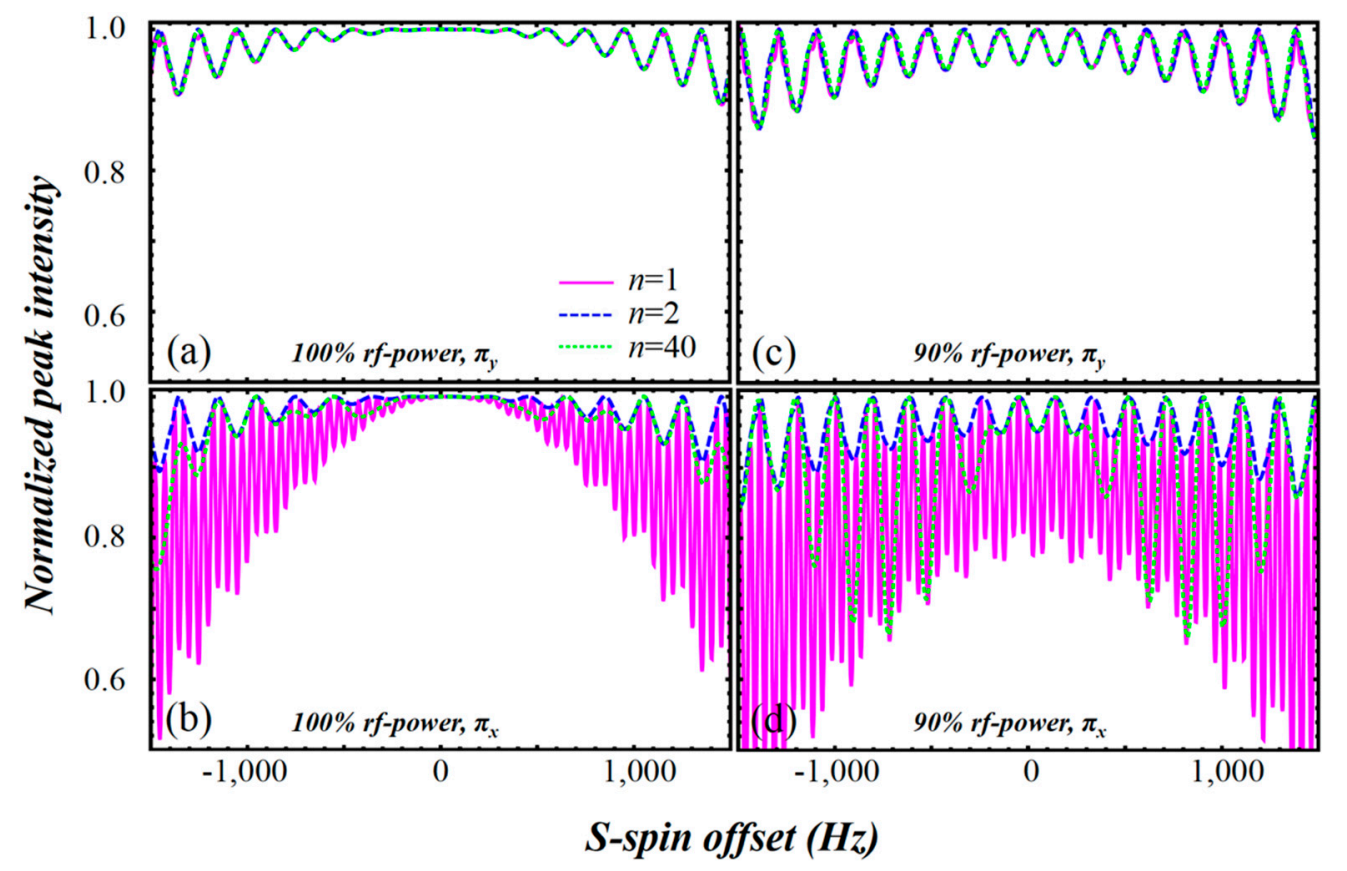

Figure 4. Cont. 


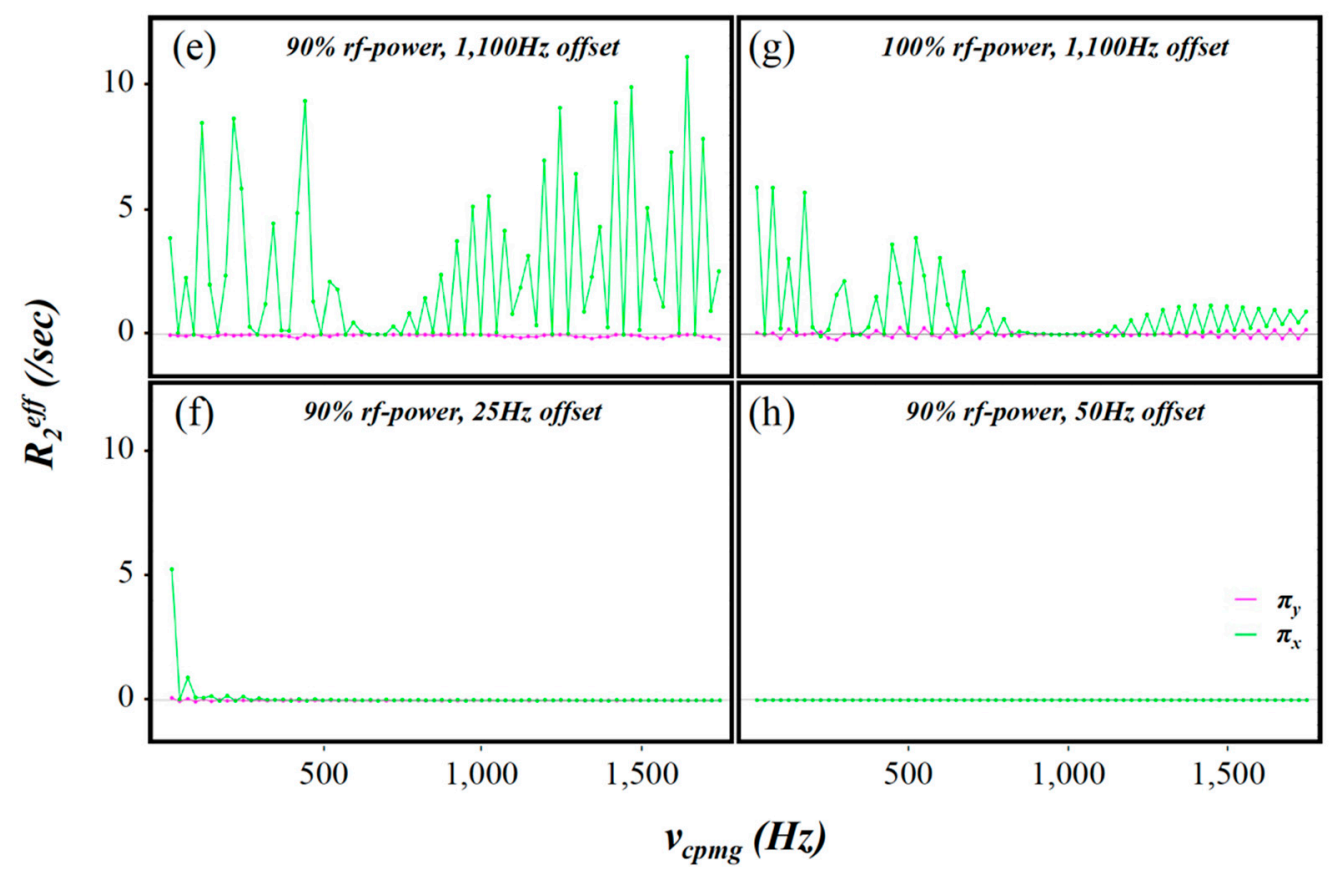

Figure 4. A simulation of artifacts occurring in rc CPMG experiments. Magnetization $2 S_{y} I_{z}$ was allowed to evolve during $C P M G_{1}$, U-element, and $C P M G_{2}$ periods. (a-d) The normalized peak intensities of the TROSY magnetization component $S_{x} I_{\beta}$ are plotted as a function of the $S$ spin resonance offset ranging from -1500 to $1500 \mathrm{~Hz}$ at intervals of $3.0 \mathrm{~Hz}$. The simulation assumed that the $S$ and $I$ spins have no intrinsic $R_{1}$ or $R_{2}$ relaxation and no exchange contribution to apparent $R_{2}$. Spin-echoes were repeated $n$ times during each CPMG constant time period of $20 \mathrm{~ms}$ (solid magenta line: $n=1$ ( $v_{\text {cpmg }}$ $=25 \mathrm{~Hz})$, dashed blue line: $n=2\left(v_{\text {cpmg }}=50 \mathrm{~Hz}\right)$, and dotted green line: $\left.n=40\left(v_{\text {cpmg }}=1000 \mathrm{~Hz}\right)\right)$. The CPMG $\pi$ pulses were applied with a width of $80 \mu$ s with a phase of $y$ and $x$ during the $C M_{1}$ and $\mathrm{CPMG}_{2}$ periods, respectively. In $(\mathbf{a}, \mathbf{b})$, the $\mathrm{rf}$ field strength for spin $S$ was set to the value exact on resonance (i.e., $6250 \mathrm{~Hz}$ ). In (c,d), the rf field strength was reduced by $10 \%$ without changing the pulse width. Thus, the timing of all pulses and delays was the same in all four cases. Furthermore, in (a,c), the phase of the $S$ spin $\pi$ pulse in the U-element was cycled as $\{y,-y\}$, and each pair of intensities was averaged. In $(\mathbf{b}, \mathbf{d})$, the corresponding phase was cycled as $\{x,-x\}$, and each pair of intensities was averaged. The pulse length and rf field strength for spin $S$ in the U-element were set to the same values as those in the CPMG periods. The ${ }^{1} \mathrm{H} \pi$ pulses in the U-element were applied with a length of $20 \mu \mathrm{s}$ with the power exact on resonance $(25 \mathrm{kHz})$. The ${ }^{1} J$ coupling constant between spins $S$ and $I$ was set to $100 \mathrm{~Hz}$. (e-h) Simulated rd curves for the rc CPMG pulse sequence with the $S$ spin $\pi$ pulse phase in the U-element set to $y$ (magenta) and $x$ (green) are compared. The magnitude of the final TROSY magnetization component $S_{x} I_{\beta}\left(I_{\text {cpmg }}\right.$ ) was calculated in the same way as in (a-d). The reference magnitude $\left(I_{\text {ref }}\right)$ was also calculated without either applied CPMG period. The effective relaxation rate $R_{2}{ }^{\text {eff }}$ was modeled as $-\left(1 / T_{\text {cpmg }}\right) \ln \left(I_{\text {cpmg }} / I_{\text {ref }}\right)$, where $T_{\text {cpmg }}$ was a total CPMG period of $40 \mathrm{ms,}$ and $R_{2}{ }^{\text {eff }}$ is plotted as a function of $v_{\text {cpmg }}(1 /(4 \delta))$. The repetition count $n$ was incremented from 0 (reference) to 70 , so that $v_{\text {cpmg }}\left(=n / T_{\text {cpmg }}\right)$ covered a range from 0 to $1750 \mathrm{~Hz}$. The $S$ spin resonance offsets were set to $1100 \mathrm{~Hz}$ in $(\mathbf{e}, \mathbf{g})$, and $25 \mathrm{~Hz}$ in (f) and $50 \mathrm{~Hz}$ in (h). In (e,f,h), the rf field strength was reduced by $10 \%$ with the pulse width unchanged, compared to the exact power in $(\mathrm{g})$. The remaining parameters were the same as those used in $(\mathbf{a}-\mathbf{d})$.

\section{Discussion}

According to the example shown in Figure 1, when the $\pi$ pulse phase was set to $y$, artifacts in the TROSY magnetization components alone reduced. Conversely, when the phase was shifted to $x$, artifacts in the anti-TROSY magnetization components were compensated, and those in the TROSY counterparts were rather accumulated. The problem that minimizing artifacts on both magnetization 
components simultaneously is basically impossible would be overcome if spin-lock pulses are applied to the amide ${ }^{1} \mathrm{H}$ spins synchronously with each ${ }^{15} \mathrm{~N}$ spin-echo to keep the in-phase ${ }^{15} \mathrm{~N}$ magnetization decoupled from the ${ }^{1} \mathrm{H}$ spins during the CPMG pulse trains [23]. However, since the ${ }^{1} \mathrm{H}$ rf field strength for the decoupling is considerably large (about $14 \mathrm{kHz}$ ) [35], care must be taken in the heating effects caused by the ${ }^{1} \mathrm{H}$ decoupling $[12,28]$. In addition, the ${ }^{1} \mathrm{H}$ decoupling rf field strength must be adjusted to be an even multiple of $v_{\text {cpmg }}$ [23].

As shown in Figure $1 \mathrm{~b}-\mathrm{g}$, the U-element inverts one of the two magnetization components of spin $S\left({ }^{15} \mathrm{~N}\right.$ or $\left.{ }^{13} \mathrm{C}\right)$ so that in the subsequent $\mathrm{CPMG}_{2}$ period, the inverted magnetization component is rotated by the $\pi$ pulses about the effective magnetic field to a direction opposite to that used in the $\mathrm{CPMG}_{1}$ period. For example, CPMG $\pi$ pulses are applied with phase $+y$ in the $\mathrm{CPMG}_{1}$ period and with phase $+x$ in the $C P M G_{2}$ period. The $+S_{y} I_{\beta}$ TROSY magnetization component at the end of the $C P M G_{1}$ period is converted to $-S_{x} I_{\beta}$ by the U-element containing one $S$ spin $\pi$ pulse of phase $y$ and two $I\left({ }^{1} \mathrm{H}\right)$-spin $\pi$ pulses. In contrast, the $-S_{y} I_{\alpha}$ anti-TROSY magnetization component is converted to $-S_{x} I_{\alpha}$, which has the same sign as the magnetization before the U-element, $-S_{y} I_{\alpha}$. The Uor P-element inverts the orientation of the TROSY magnetization vector almost completely around the axis orthogonal to the effective magnetic field, as shown in Figure 5. Consequently, the TROSY magnetization components that are wound about the effective magnetic field during the $\pi$ pulses and about the $z$-axis during delays $\delta$ in the $C P M G_{1}$ period are subsequently rewound about those in the $C P M G_{2}$ period. Most unwanted magnetization that is generated by pulse imperfections in the $C P M G_{1}$ period are refocused by those in the $C P M G_{2}$ period. For the anti-TROSY magnetization components, in contrast, the artifacts generated in the $C P M G_{1}$ period are not canceled in the $C P G_{2}$ period. Instead, they are rather accumulated because the magnetization vectors are not inverted by the U- or P-element, i.e., the vectors remain oriented in almost the same direction with respect to the effective magnetic field with the signs of the coherences unchanged before and after the U-element. The U-element having the $\pi$ pulse phase shifted by $90^{\circ}$ from $+y$ to $+x$ converts the TROSY $\left(+S_{y} I_{\beta}\right)$ and anti-TROSY $\left(-S_{y} I_{\alpha}\right)$ magnetization components to $+S_{x} I_{\beta}$ and $+S_{x} I_{\alpha}$, respectively. As a result, the TROSY magnetization component vectors are then rotated to the same direction about the effective magnetic fields for both CPMG periods with artifacts accumulating, while artifacts in the anti-TROSY magnetization components are refocused.

Whether the U- or P-element actually inverts the TROSY magnetization component vector depends on the relationship between the sign of the associated ${ }^{1} J$ coupling constant and the phases of the CPMG refocusing pulses, which may also depend on the NMR machines used. We optimized the phases using Bruker spectrometers. In Varian spectrometers, however, the effective sign of phase $y$ in the $\mathrm{CPMG}_{1}$ period is likely opposite to that of Bruker ones [36]. In this case, artifacts are canceled in the TROSY magnetization component when the central $\pi$ pulse phase is set to $x$. This was confirmed in our simulation. Even if a pulse program that is faithfully translated is used and appropriate TROSY correlation peaks are observed in the multiplet components in spectra, the desired TROSY magnetization component may not be the subject of artifact suppression. We suggest that a pair of $2 \mathrm{D}$ spectra $(n=1)$ be measured with the central $\pi$ pulse phase set to $x$ and $y$ using a concentrated sample $(>500 \mu \mathrm{L})$ and that the peak intensities be compared once before the first use of pulse programs.

In addition to the U-element, Vallurupalli et al. proposed the P-element, which has two ${ }^{15} \mathrm{~N} \pi / 2$ pulses enclosing the U-element [21]. The U- and P-elements are nearly the same, except for a slight difference described below. As shown in Figure 5, both elements invert one of the two magnetization components, e.g., TROSY of spin $S$ in a spin-state selective manner. The inversion is almost complete as far as the pulses in the P-element have no imperfection. In contrast, there is a difference in the behavior of the other magnetization component, e.g., anti-TROSY, depending on which element, P or $\mathrm{U}$, is used. 

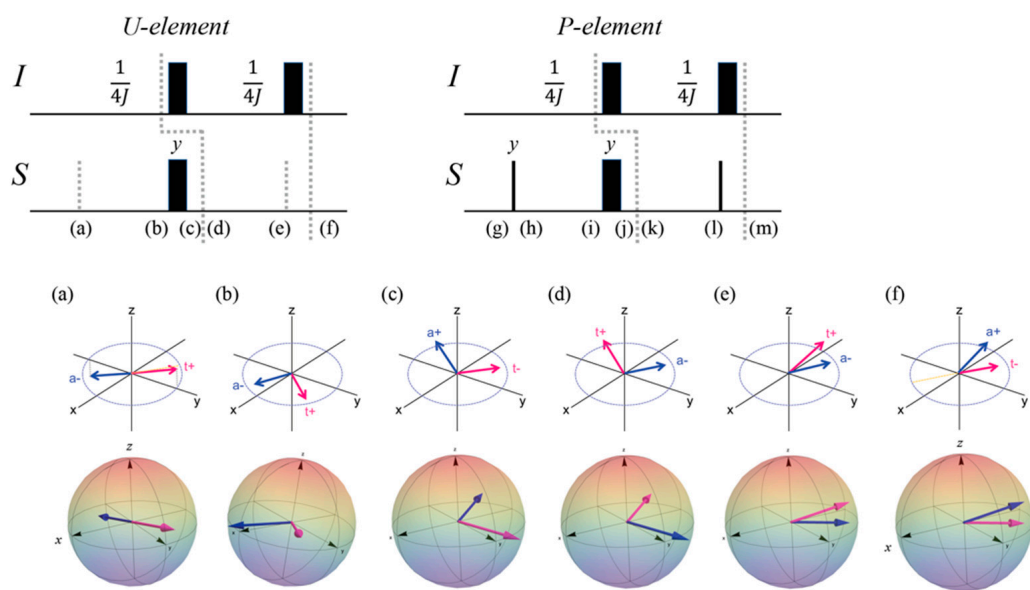

(g)

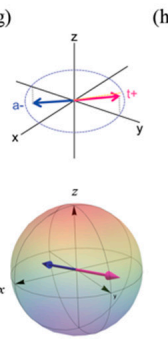

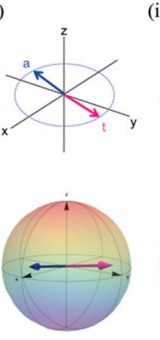
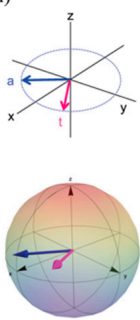

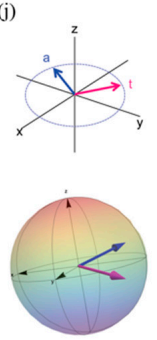

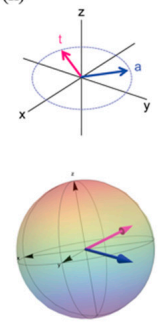

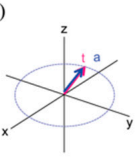

(m)

Figure 5. Behaviors of magnetization vectors in the U-element (a-f) and P-element (g-m) periods in rc CPMG rd experiments, shown by a vector model in the rotating coordinate system. The vectors of the TROSY $S_{y} I_{\beta}$ and anti-TROSY $-S_{y} I_{\alpha}$ magnetization components are indicated by red and blue arrows, respectively. The signs " + " and "-" indicate that the corresponding vector is above and below the $x-y$ transverse plane, respectively. It is assumed that the initial magnetization $-2 S_{y} I_{z}$ already contain errors that has accumulated during the previous $C \mathrm{PMG}_{1}$ period. The two vectors representing $-2 S_{y} I_{z}$ are initially directed along orientations deviating from the $\pm y$ axis by $(\theta, \phi)=(10,10)$ for $S_{y} I_{\beta}$ and by $(\theta, \phi)=(10,-10)$ for $-S_{y} I_{\alpha}$ in $(\mathbf{a}, \mathbf{g})$, where $\theta$ and $\phi$ represent the azimuth and altitude angles in a polar coordinate system, respectively. The evolution of spin $S$ by ${ }^{1}{ }_{\text {IS }}$ coupling is included during the total delay $1 /\left(2^{1} J\right)$, but the chemical shift evolution of spin $S$ is removed.

According to simulations with various parameters, large artifacts were found in the anti-TROSY magnetization component at a regular interval of $1 /(2 \delta) \mathrm{Hz}$ in a plot of the signal intensity as a function of the chemical shift frequency. This is particularly noticeable when odd numbers of spin-echoes were implemented in each CPMG period. Ishima also describes that off resonance-caused errors maximize in CPMG $R_{2}$ measurements when the difference between the signal and carrier frequencies equals $k /(2 \delta)$, where $k$ is an integer [37]. Our simulations also showed that all the artifact maxima shifted constantly by $+\mathrm{J} / 2 \mathrm{~Hz}$. The results indicate that errors maximize when the anti-TROSY magnetization vector, $S_{y} I_{\alpha}$, becomes parallel to the phase axis $y$ of the CPMG refocusing $\pi$ pulses at the moment the pulses are applied. In such a situation, the effective magnetic field rotates the magnetization vector that is almost parallel to the phase axis to a position that deviates from the transverse $x-y$ plane in the rotating coordinate frame (Figure $6 \mathrm{a}, \mathrm{b}$ ). This result was also confirmed by a simpler simulation, where the initial magnetization $S_{y}$ experienced a series of $n$ spin-echoes when it was not coupled to any other spins. Each $\pi$ pulse of phase $y$ was placed in the middle of a period $2 \delta$ (i.e., $\left\{\delta-\pi_{y}-\delta\right\}_{n}$ ), and was applied with a power exact on resonance $(80 \mu \mathrm{s})$. The final $S_{y}$ intensity was calculated and plotted against the $S$ spin chemical shift. With the off-resonance effects occurring, error maxima were found at an offset of $k /(2 \delta)$ (= even multiples of $v_{\text {cpmg }}$ ), particularly when $n$ was an odd number (Figure $7 \mathrm{a}, \mathrm{c}$ ). Since the magnetization vector cycled around the $z$-axis $k$ times during the inter-pulse delay of $2 \delta$, most errors were canceled after even-numbered spin-echoes. In contrast, when the rf field strength 
of the $\pi$ pulses was reduced by $10 \%$ than the exact value and the off-resonance effect was removed during pulse implementation, error maxima were found at an offset of $k /(2 \delta)+1 /(4 \delta)(=$ odd multiples of $v_{\text {cpmg }}$ ), particularly when $n$ was an odd number (Figure $4 \mathrm{f}, \mathrm{h}$ and Figure $7 \mathrm{~b}, \mathrm{~d}$ ). This result indicates that pulse miscalibration generates large artifacts when the magnetization vector comes at right angles $x$ to the phase axis $y$ of the CPMG $\pi$ pulses at the moment the pulses are applied. In such a case, the magnetization vector rotates about the $z$-axis by $(2 k+1) \pi$ radian during the inter-pulse delay of $2 \delta$ (Figure $6 \mathrm{c}, \mathrm{d}$ ). As a consequence, the deviation of the vector from the $x$-axis is almost compensated after even-numbered spin-echoes. As Ishima pointed out [37], when $2 \delta$ is set to $1 \mathrm{~ms}$, as is often adopted in ${ }^{15} \mathrm{~N} R_{2}$ CPMG relaxation measurements, the first of the cyclic artifact surges appears at $\pm 1000 \mathrm{~Hz}$ offset, which is almost outside a typical spectral region for main-chain amide ${ }^{15} \mathrm{~N}$ spins at a static magnetic field of $600 \mathrm{MHz}$. In rc CPMG rd experiments, however, $2 \delta$ extends to a period as long as $20 \mathrm{~ms}$ (e.g., a total relaxation delay of $40 \mathrm{~ms}$ and $n=1$ ). This leads to large artifacts occurring every $50 \mathrm{~Hz}$. This explains the reason that artifacts are most often encountered at the lowest $v_{\text {cpmg }}(n=1$, odd). Artifacts appear even in peaks resonating at an offset close to the $S$ spin carrier frequency. Since the artifact intensity oscillates along the offset frequency at a cycle that depends on $\delta$, peak intensities measured with a series of various lengths of $\delta$ carry artifacts of $\delta$-dependent intensities, unlike $T_{2}$ relaxation measurements that use a constant delay $\delta$ (e.g., $500 \mu \mathrm{s}$ ) [22]. It can also be easily explained why we observed a drastic change in the overall appearance of error peaks by shifting the ${ }^{15} \mathrm{~N}$ carrier frequency by just $1.0 \mathrm{ppm}$ under a ${ }^{1} \mathrm{H}$ static magnetic field of $800 \mathrm{MHz}$ (Figure 2d).

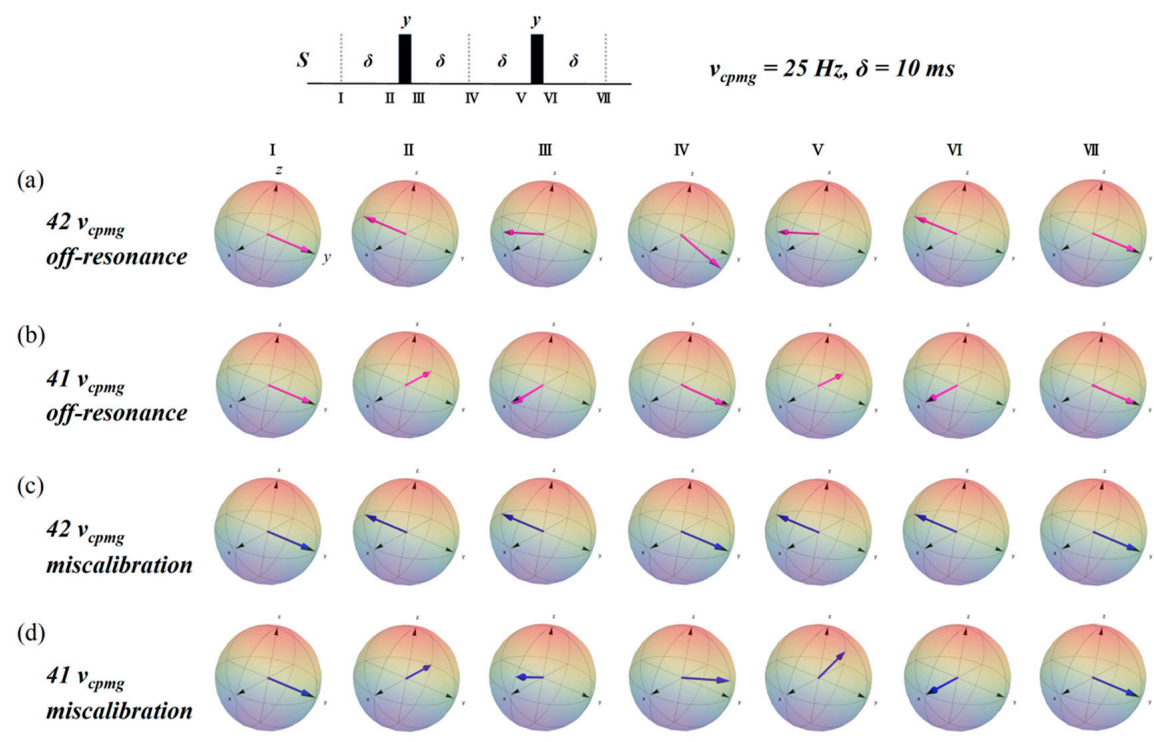

Figure 6. Magnetization vectors sampled at time points designated as (I-VII) in a simple CPMG pulse sequence, shown by a vector model in the rotating coordinate system. Two spin-echoes $\left\{\delta-\pi_{y}-\delta\right\}_{2}$ were applied to the initial magnetization $S_{y}$, which had no $J$ coupling to any other spins. The period $\delta$ was set to $10 \mathrm{~ms}\left(v_{\mathrm{cpmg}}=1 /(4 \delta)=25 \mathrm{~Hz}\right)$. The parameters used were almost the same as those in Figure 7 . The refocusing $\pi$ pulses were applied along the $y$-axis with a length of $80 \mu \mathrm{s}$. $(\mathbf{a}, \mathbf{b})$ show simulation results involving off-resonance effects during the application of exactly calibrated pulses. The rf field strength was set to $6250 \mathrm{~Hz}$. (c,d) show the results of simulation in which the refocusing $\pi$ pulses were applied with the rf field strength reduced by $10 \%$ from the exact power with no off-resonance effects occurring during pulse application. The spin $S$ frequency offsets were set to $42 * v_{\text {cpmg }}=1050 \mathrm{~Hz}$ in $(\mathbf{a}, \mathbf{c})$ and to $41^{*} v_{\mathrm{cpmg}}=1025 \mathrm{~Hz}$ in $(\mathbf{b}, \mathbf{d})$. As shown in (a, IV), large deviations from the ideal orientation were found in the spin $S$ vector at the frequency offsets of even multiples of $v_{\text {cpmg. Such }}$ deviations occurred after an odd number of spin-echoes even if the pulse power was exactly calibrated. In contrast, pulse miscalibration caused large errors at the frequency offsets of odd multiples of $v_{\text {cpmg }}$ after odd-numbered spin-echoes, as shown in (d, IV). 
The standard pulse program library of Bruker includes a rc CPMG rd pulse program of the ${ }^{1} \mathrm{H} /{ }^{15} \mathrm{~N}$ TROSY version (trrexetf3gpsi3d). It is, however, different from the one described above in that the part corresponding to the U-element contains only one $\pi$ pulse for the ${ }^{1} \mathrm{H}^{\mathrm{N}}$ spins, i.e., $1 /\left(4^{1} J_{\mathrm{HN}}\right)-\pi^{\mathrm{H}} / \pi^{\mathrm{N}}-1 /\left(4^{1} J_{\mathrm{HN}}\right)$. Although the program is designed so that the part following the pair of CPMG pulse trains selects the TROSY magnetization components of ${ }^{15} \mathrm{~N}$ and ${ }^{1} \mathrm{H}$ spins, its U-element switches between the ${ }^{15} \mathrm{~N}$ TROSY and anti-TROSY magnetization components. Therefore, the ${ }^{15} \mathrm{~N}$ anti-TROSY magnetization component that mixes in the detected ${ }^{1} \mathrm{H} /{ }^{15} \mathrm{~N}$ TROSY signals lowers the rd sensitivity.

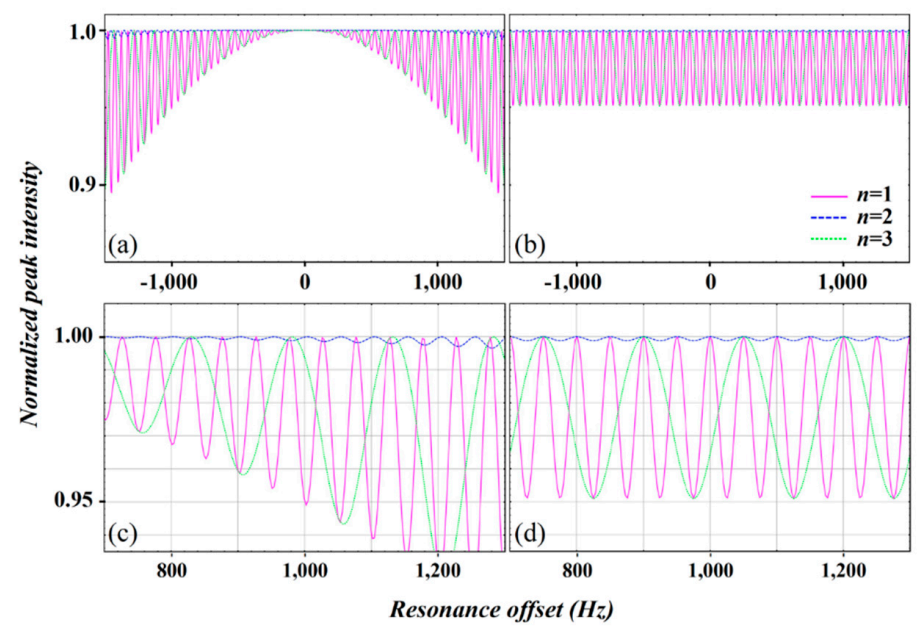

Figure 7. Simulated artifacts occurring in a simple CPMG pulse sequence. A series of $n$-repeated spin-echoes, $\left\{\delta-\pi_{y}-\delta\right\}_{n}$, were applied within a constant-time period of $20 \mathrm{~ms}$ to magnetization $S_{y}$, which had no $J$ coupling to any other spins. The final intensity of $S_{y}$ is plotted as a function of the resonance offset ranging from -1500 to $1500 \mathrm{~Hz}$ at intervals of $3.0 \mathrm{~Hz}$. (a) The refocusing $\pi$ pulses were applied along the $y$-axis with a duration of $80 \mu$ s and the exact rf field strength $(6250 \mathrm{~Hz})$. Thus, the simulation involved off-resonance effects without pulse miscalibration; (b) Refocusing $\pi$ pulses were applied with the rf field strength reduced by $10 \%$ from the exact value, with no

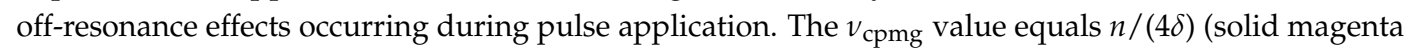
line: $n=1\left(v_{\text {cpmg }}=25 \mathrm{~Hz}\right)$, dashed blue line: $n=2\left(v_{\text {cpmg }}=50 \mathrm{~Hz}\right)$, and dotted green line: $n=3\left(v_{\mathrm{cpmg}}\right.$ $=75 \mathrm{~Hz}))$. In (a), the error maxima were found at frequency offsets that were even multiples of $v_{\text {cpmg }}$, while in (b) they were found at frequency offsets that were odd multiples of $v_{\text {cpmg. This indicates }}$ that errors accumulate in $(\mathbf{a}, \mathbf{b})$ in the cases where the magnetization vector aligns along the $y$ and $x$ axes, respectively, after $\delta$ and just before the first $\pi$ pulse of phase $y$. These errors are compensated when the vector returns back to almost the same position along the $y$ and $x$ axes, respectively, after $\left\{\delta-\pi_{y}-\delta-\delta\right\}$ and experiences the second $\pi$ pulse of phase $y$. Such error accumulation and compensation occur alternately after every odd and even numbered spin-echo, respectively. The offset frequencies at which the changes maximize vary depending on the causes of errors. The off-resonance effects and pulse miscalibration generate maximum errors at offset frequencies of even and odd multiples of $v_{\text {cpmg, }}$, respectively. Parts of $(\mathbf{a}, \mathbf{b})$ are enlarged and shown in $(\mathbf{c}, \mathbf{d})$, respectively. The corresponding vector models are depicted in Figure 6.

\section{Materials and Methods}

\subsection{Sample Preparation and NMR Measurements}

For amide ${ }^{15} \mathrm{~N}$ spin rd experiments, a protein sample of the chitin-binding domain of chitinase A1 (ChiA1) from Bacillus circulans WL-12 (PDB code: 1ED7), constituted of 45 amino acids, was used. A previous measurement and subsequent model-free analysis of ${ }^{15} \mathrm{~N}$ spin longitudinal $\left(T_{1}\right)$ and transverse $\left(T_{2}\right)$ relaxation times and steady-state heteronuclear NOE showed that ChiA1 has 
no significant conformational exchange with a rate constant $\left(k_{\mathrm{ex}}\right)$ slower than $3500 \mathrm{~s}^{-1}$ [24]. Hence, the obtained rd profiles should be almost constant against $v_{\mathrm{cpmg}}$ if the data were free of any artifacts. ChiA1 was purified through chitin-affinity, hydroxyapatite, and gel-filtration column chromatography, as described [24]. ChiA1 labeled with ${ }^{15} \mathrm{~N}$ was dissolved at a concentration of $0.2 \mathrm{mM}$ in $50 \mathrm{mM}$ potassium phosphate buffer ( $\mathrm{pH}$ 6.0) containing $5 \mathrm{mM}$ deuterated DTT and $10 \% \mathrm{D}_{2} \mathrm{O}$. The sample $(600 \mu \mathrm{L})$ was packed into a normal NMR sample tube, not into a Shigemi tube suitable for a small volume. The large volume was expected to increase the effect from the $B_{1}$ field inhomogeneity, i.e., the pulse imperfection. NMR experiments were conducted with the pulse sequence shown in Figure 1a at $293 \mathrm{~K}$ using NMR spectrometers with ${ }^{1} \mathrm{H}$ basic resonance frequencies of 500.13 and $800.23 \mathrm{MHz}$ (BrukerBioSpin Avance III HD with TCI cryogenic probes). CPMG $\pi$ pulses were applied to ${ }^{15} \mathrm{~N}$ spins for a total constant relaxation time of $40 \mathrm{~ms}$ with repetition rates $(1 /(4 \delta))$ of 0 (reference), 25, 200, 350, $525,675,850$, and $1000 \mathrm{~Hz}$ in a rc manner without ${ }^{1} \mathrm{H}$ decoupling by continuous-wave or composite pulses $[15,21]$. The CPMG ${ }^{15} \mathrm{~N} \pi$ pulse lengths were set at 92 and $80 \mu$ s for the 500 and $800 \mathrm{MHz}$ spectrometers, respectively. The spectral widths (the number of total data points) on the $500 \mathrm{MHz}$ spectrometer were $24 \mathrm{ppm}$ (2048) for the ${ }^{1} \mathrm{H}$ dimension, and $35 \mathrm{ppm}$ (200) for the ${ }^{15} \mathrm{~N}$ dimension. Those on the $800 \mathrm{MHz}$ spectrometer were $16 \mathrm{ppm}$ (2048) for the ${ }^{1} \mathrm{H}$ dimension, and $35 \mathrm{ppm}$ (180) for the ${ }^{15} \mathrm{~N}$ dimension. Sixteen scans were accumulated for each free induction decay (FID). The carrier frequencies were placed at 4.7 and $119 \mathrm{ppm}$ for the ${ }^{1} \mathrm{H}$ and ${ }^{15} \mathrm{~N}$ dimensions, respectively. A relaxation delay between successive scans was set at $2.8 \mathrm{~s}$.

For aromatic ${ }^{13} \mathrm{C}$ spin rd experiments, alternate ${ }^{13} \mathrm{C}$-labeled and uniformly ${ }^{15} \mathrm{~N}$-labeled ChiA 1 was produced by growing Escherichia coli in an M9 medium containing $2.0 \mathrm{~g} / \mathrm{L}\left[2-{ }^{13} \mathrm{C}\right]$-glucose and $1.0 \mathrm{~g} / \mathrm{L}^{15} \mathrm{NH}_{4} \mathrm{Cl}[30,33]$. The protein was dissolved at a concentration of $0.3 \mathrm{mM}$ in $20 \mathrm{mM}$ deuterated acetic acid buffer $\left(\mathrm{CD}_{3} \mathrm{COONa}\right)(\mathrm{pH} 4.0)$ containing $90 \% \mathrm{D}_{2} \mathrm{O}$. Ten-percent ${ }^{1} \mathrm{H}_{2} \mathrm{O}$ contained in the sample enabled us to run a three-dimensional gradient shimming (Topshim 3D) and to confirm that the sample did not degrade through $2 \mathrm{D}^{1} \mathrm{H} /{ }^{15} \mathrm{~N}$ HSQC spectra. NMR experiments were conducted at $298 \mathrm{~K}$. The $\mathrm{CPMG}{ }^{13} \mathrm{C} \pi$ pulse lengths were set at 50 and $40 \mu$ s for the 500 and $800 \mathrm{MHz}$ spectrometers, respectively. The spectral widths (the number of total data points) on the $500 \mathrm{MHz}$ spectrometer were $28 \mathrm{ppm}$ (2048) for the ${ }^{1} \mathrm{H}$ dimension, and $30 \mathrm{ppm}(60)$ for the ${ }^{13} \mathrm{C}$ dimension. Those on the $800 \mathrm{MHz}$ spectrometer were $17 \mathrm{ppm}$ (2048) for the ${ }^{1} \mathrm{H}$ dimension, and $30 \mathrm{ppm}$ (128) for the ${ }^{13} \mathrm{C}$ dimension. Forty-eight scans were accumulated for each free induction decay (FID). The carrier frequencies were placed at 4.7 and $125 \mathrm{ppm}$ for the ${ }^{1} \mathrm{H}$ and ${ }^{13} \mathrm{C}$ dimensions, respectively. The other parameters were the same as those for the amide ${ }^{15} \mathrm{~N}$ rd experiments.

All data sets were processed and analyzed with the NMRPipe [38] program. The ${ }^{15} \mathrm{~N}$ and ${ }^{13} \mathrm{C}$ pulse field strengths were calibrated by $1 \mathrm{D}$ versions of modified ${ }^{1} \mathrm{H} /{ }^{15} \mathrm{~N}$ HSQC and HNCO experiments, respectively, with a precision of about $0.1 \mu$ s every time the sample was set in NMR machines. In the modified HNCO experiment, $\mathrm{a}^{13} \mathrm{C}^{\prime} \pi / 2$ pulse to be adjusted was included instead of the ${ }^{13} \mathrm{C}$ chemical shift evolution period. All the ${ }^{13} \mathrm{C}^{\prime} \pi$ pulses were applied as ${ }^{13} \mathrm{C}^{\prime}$-selective adiabatic pulses whose performance is tolerant of a small deviation from the still unknown exact power. We used no parameters related to ${ }^{15} \mathrm{~N}$ or ${ }^{13} \mathrm{C}$ pulse widths that were determined previously with $\left[{ }^{15} \mathrm{~N}\right]$-urea or $\left[{ }^{13} \mathrm{C}\right]$-methanol to minimize the contribution of ${ }^{15} \mathrm{~N} /{ }^{13} \mathrm{C}$ pulse miscalibrations to artifacts.

\subsection{Simulation}

The magnetization behaviors in CPMG pulse sequences were simulated with in-house software written in Mathematica (Wolfram). The program included the evolution by the chemical shift and $J$ coupling during the pulse application periods. We set the pulse lengths in the program to the same values as were used in the actual NMR experiments. Bloch equations of a J-coupled heteronuclear IS two-spin system were solved to calculate each coherence magnitude. No relaxation or exchange was included in the simulation. 


\section{Conclusions}

The combination of our NMR experiments and simulation showed that particularly at the lowest $v_{\text {cpmg, }}$ large artifacts can appear even in amide ${ }^{1} \mathrm{H}-{ }^{15} \mathrm{~N}$ and aromatic ${ }^{1} \mathrm{H}-{ }^{13} \mathrm{C}$ correlation peaks that are very close to the ${ }^{15} \mathrm{~N} /{ }^{13} \mathrm{C}$ carrier frequencies. The numerical and vector model simulation showed that the off-resonance effects and miscalibration of the CPMG $\pi$ pulses generate artifacts whose magnitudes oscillate along the resonance offset with maxima at offsets equal to even and odd multiples of $v_{\mathrm{cpmg}}$, respectively. Since the lowest $v_{\text {cpmg }}$ reaches as small as $25 \mathrm{~Hz}$, artifacts appear at offsets of every several tens of hertz and disappear with the carrier frequency shifted by less than $1 \mathrm{ppm}$, as demonstrated in NMR experiments. In addition, it is well known that even numbers of consecutive CPMG $\pi$ pulses have a property of compensating for artifacts. These explain why artifacts are most often seen when a single spin-echo is placed in each CPMG period, namely, at the lowest $v_{\text {cpmg }}$. The fact that the resonance offsets at which artifacts maximize depend on $v_{\text {cpmg }}$ also explains a reason that rd experiments are vulnerable to artifacts compared to $R_{2}$ relaxation measurement experiments, which use a constant inter- $\pi$ pulse delay of about $1 \mathrm{~ms}$. We also demonstrate that artifacts are significantly suppressed in TROSY-based rc CPMG rd experiments when the ${ }^{15} \mathrm{~N} /{ }^{13} \mathrm{C} \pi$ pulse phase in the U- or P-element is properly chosen between $x$ and $y$. Which phase leads to the artifact cancellation may depend on the spectrometer design. Pulse sequences with the proper phase are tolerant to a deviation of the CPMG $\pi$ pulse rf power as large as $\pm 10 \%$ within the spectral ranges of most mainchain amide ${ }^{15} \mathrm{~N}$ and aromatic ${ }^{13} \mathrm{C}$ spins of proteins.

Author Contributions: T.I. and T.K. conceived and designed the experiments; T.K. and J.K. performed the experiments and analyzed the data; T.K. and A.N. contributed preparation of samples; T.I. wrote the paper.

Funding: This study was funded by JSPS KAKENHI (Grant-in-Aid for Challenging Exploratory Research) Grant Number 16K14683 and a Grant for academic research from Yokohama City University.

Acknowledgments: T.I. appreciates Shota Motoyama helping prepare the samples, the Yoshifumi Nishimura's group members maintaining the NMR equipment and Profs. Hayuki Sugimoto and Takeshi Watanabe in Niigata University discussing the sample preparation.

Conflicts of Interest: The authors declare no conflict of interest.

\section{References}

1. Akke, M.; Palmer, A.G. Monitoring macromolecular motions on microsecond to millisecond time scales by $\mathrm{R}_{1 \rho}-\mathrm{R}_{1}$ constant relaxation time NMR spectroscopy. J. Am. Chem. Soc. 1996, 118, 911-912. [CrossRef]

2. Mulder, F.A.; Mittermaier, A.; Hon, B.; Dahlquist, F.W.; Kay, L.E. Studying excited states of proteins by NMR spectroscopy. Nat. Struct. Biol. 2001, 8, 932-935. [CrossRef] [PubMed]

3. Sugase, K.; Dyson, H.J.; Wright, P.E. Mechanism of coupled folding and binding of an intrinsically disordered protein. Nature 2007, 447, 1021-1025. [CrossRef] [PubMed]

4. Sauerwein, A.C.; Hansen, D.F. Relaxation Dispersion NMR Spectroscopy. In Protein NMR: Biological Magnetic Resonance; Springer: Boston, MA, USA, 2015; pp. 75-132. ISBN 978-1-4899-7620-8.

5. Smith, C.A.; Ban, D.; Pratihar, S.; Giller, K.; Schwiegk, C.; de Groot, B.L.; Becker, S.; Griesinger, C.; Lee, D. Population shuffling of protein conformations. Angew. Chem. Int. Ed. Engl. 2015, 54, 207-210. [CrossRef] [PubMed]

6. Pratihar, S.; Sabo, T.M.; Ban, D.; Fenwick, R.B.; Becker, S.; Salvatella, X.; Griesinger, C.; Lee, D. Kinetics of the antibody recognition site in the third IgG-binding domain of protein G. Angew. Chem. Int. Ed. Engl. 2016, 55, 9567-9570. [CrossRef] [PubMed]

7. Ban, D.; Smith, C.A.; de Groot, B.L.; Griesinger, C.; Lee, D. Recent advances in measuring the kinetics of biomolecules by NMR relaxation dispersion spectroscopy. Arch. Biochem. Biophys. 2017, 628, 81-91. [CrossRef] [PubMed]

8. Ikeya, T.; Ban, D.; Lee, D.; Ito, Y.; Kato, K.; Griesinger, C. Solution NMR views of dynamical ordering of biomacromolecules. Biochim. Biophys. Acta 2018, 1862, 287-306. [CrossRef] [PubMed] 
9. Korzhnev, D.M.; Orekhov, V.Y.; Dahlquist, F.W.; Kay, L.E. Off-resonance $R_{1} \rho$ relaxation outside of the fast exchange limit: An experimental study of a cavity mutant of T4 lysozyme. J. Biomol. NMR 2003, 26, 39-48. [CrossRef] [PubMed]

10. Carr, H.Y.; Purcell, E.M. Effects of diffusion on free precession in nuclear magnetic resonance experiments. Phys. Rev. 1954, 94, 630-638. [CrossRef]

11. Meiboom, S.; Gill, D. Modified spin-echo method for measuring nuclear relaxation times. Rev. Sci. Instrum. 1958, 29, 688-691. [CrossRef]

12. Gopalan, A.B.; Hansen, D.F.; Vallurupalli, P. CPMG Experiments for Protein Minor Conformer Structure Determination. In Protein NMR: Methods in Molecular Biology; Humana Press: New York, NY, USA, 2018; pp. 223-242. ISBN 978-1-4939-7385-9.

13. Chatterjee, S.D.; Ubbink, M.; van Ingen, H. Removal of slow-pulsing artifacts in in-phase ${ }^{15} \mathrm{~N}$ relaxation dispersion experiments using broadband ${ }^{1} \mathrm{H}$ decoupling. J. Biomol. NMR 2018, 71, 69-77. [CrossRef] [PubMed]

14. Loria, J.P.; Rance, M.; Palmer, A.G. A relaxation-compensated Carr-Purcell-Meiboom-Gill sequence for characterizing chemical exchange by NMR spectroscopy. J. Am. Chem. Soc. 1999, 121, 2331-2332. [CrossRef]

15. Loria, J.P.; Rance, M.; Palmer, A.G. A TROSY CPMG sequence for characterizing chemical exchange in large proteins. J. Biomol. NMR 1999, 15, 151-155. [CrossRef] [PubMed]

16. Ghose, R. Average Liouvillian theory in nuclear magnetic resonance-Principles, properties, and applications. Concepts Magn. Reson. 2000, 12, 152-172. [CrossRef]

17. Sørensen, M.D.; Meissner, A.; Sørensen, O.W. Spin-state-selective coherence transfer via intermediate states of two-spin coherence in IS spin systems: Application to E.COSY-type measurement of J coupling constants. J. Biomol. NMR 1997, 10, 181-186. [CrossRef]

18. Pervushin, K.; Riek, R.; Wider, G.; Wüthrich, K. Attenuated $\mathrm{T}_{2}$ relaxation by mutual cancellation of dipole-dipole coupling and chemical shift anisotropy indicates an avenue to NMR structures of very large biological macromolecules in solution. Proc. Natl. Acad. Sci. USA 1997, 94, 12366-12371. [CrossRef] [PubMed]

19. Waluch, V.; Bradley, W.G. NMR even echo rephasing in slow laminar flow. J. Comput. Assist. Tomogr. 1984, 8, 594-598. [CrossRef] [PubMed]

20. Tollinger, M.; Skrynnikov, N.R.; Mulder, F.A.; Forman-Kay, J.D.; Kay, L.E. Slow dynamics in folded and unfolded states of an SH3 domain. J. Am. Chem. Soc. 2001, 123, 11341-11352. [CrossRef] [PubMed]

21. Vallurupalli, P.; Hansen, D.F.; Stollar, E.; Meirovitch, E.; Kay, L.E. Measurement of bond vector orientations in invisible excited states of proteins. Proc. Natl. Acad. Sci. USA 2007, 104, 18473-18477. [CrossRef] [PubMed]

22. Mandel, A.M.; Akke, M.; Palmer, A.G. Backbone dynamics of Escherichia coli ribonuclease HI: Correlations with structure and function in an active enzyme. J. Mol. Biol. 1995, 246, 144-163. [CrossRef] [PubMed]

23. Hansen, D.F.; Vallurupalli, P.; Kay, L.E. An improved ${ }^{15} \mathrm{~N}$ relaxation dispersion experiment for the measurement of millisecond time-scale dynamics in proteins. J. Phys. Chem. B 2008, 112, 5898-5904. [CrossRef] [PubMed]

24. Ikegami, T.; Okada, T.; Hashimoto, M.; Seino, S.; Watanabe, T.; Shirakawa, M. Solution structure of the chitin-binding domain of Bacillus circulans WL-12 chitinase A1. J. Biol. Chem. 2000, 275, 13654-13661. [CrossRef] [PubMed]

25. Yip, G.N.B.; Zuiderweg, E.R.P. A phase cycle scheme that significantly suppresses offset-dependent artifacts in the $R_{2}-\mathrm{CPMG}^{15} \mathrm{~N}$ relaxation experiment. J. Magn. Reson. 2004, 171, 25-36. [CrossRef] [PubMed]

26. Long, D.; Liu, M.; Yang, D. Accurately probing slow motions on millisecond timescales with a robust NMR relaxation experiment. J. Am. Chem. Soc. 2008, 130, 17629. [CrossRef] [PubMed]

27. Myint, W.; Cai, Y.; Schiffer, C.A.; Ishima, R. Quantitative comparison of errors in ${ }^{15} \mathrm{~N}$ transverse relaxation rates measured using various CPMG phasing schemes. J. Biomol. NMR 2012, 53, 13-23. [CrossRef] [PubMed]

28. Reddy, J.G.; Pratihar, S.; Ban, D.; Frischkorn, S.; Becker, S.; Griesinger, C.; Lee, D. Simultaneous determination of fast and slow dynamics in molecules using extreme CPMG relaxation dispersion experiments. J. Biomol. NMR 2018, 70, 1-9. [CrossRef] [PubMed]

29. Pervushin, K.; Riek, R.; Wider, G.; Wüthrich, K. Transverse relaxation-optimized spectroscopy (TROSY) for NMR studies of aromatic spin systems in ${ }^{13}$ C-labeled proteins. J. Am. Chem. Soc. 1998, 120, 6394-6400. [CrossRef] 
30. Weininger, U.; Respondek, M.; Akke, M. Conformational exchange of aromatic side chains characterized by L-optimized TROSY-selected ${ }^{13}$ C CPMG relaxation dispersion. J. Biomol. NMR 2012, 54, 9-14. [CrossRef] [PubMed]

31. Weininger, U.; Diehl, C.; Akke, M. ${ }^{13} \mathrm{C}$ relaxation experiments for aromatic side chains employing longitudinal- and transverse-relaxation optimized NMR spectroscopy. J. Biomol. NMR 2012, 53, 181-190. [CrossRef] [PubMed]

32. Weininger, U.; Brath, U.; Modig, K.; Teilum, K.; Akke, M. Off-resonance rotating-frame relaxation dispersion experiment for ${ }^{13} \mathrm{C}$ in aromatic side chains using L-optimized TROSY-selection. J. Biomol. NMR 2014, 59, 23-29. [CrossRef] [PubMed]

33. Lundström, P.; Teilum, K.; Carstensen, T.; Bezsonova, I.; Wiesner, S.; Hansen, D.F.; Religa, T.L.; Akke, M.; Kay, L.E. Fractional ${ }^{13} \mathrm{C}$ enrichment of isolated carbons using $\left[1-{ }^{13} \mathrm{C}\right]-$ or $\left[2-{ }^{13} \mathrm{C}\right]$-glucose facilitates the accurate measurement of dynamics at backbone $\mathrm{C}^{\alpha}$ and side-chain methyl positions in proteins. J. Biomol. NMR 2007, 38, 199-212. [CrossRef] [PubMed]

34. Teilum, K.; Brath, U.; Lundström, P.; Akke, M. Biosynthetic ${ }^{13} \mathrm{C}$ labeling of aromatic side chains in proteins for NMR relaxation measurements. J. Am. Chem. Soc. 2006, 128, 2506-2507. [CrossRef] [PubMed]

35. Jiang, B.; Yu, B.; Zhang, X.; Liu, M.; Yang, D. A ${ }^{15} \mathrm{~N}$ CPMG relaxation dispersion experiment more resistant to resonance offset and pulse imperfection. J. Magn. Reson. 2015, 257, 1-7. [CrossRef] [PubMed]

36. Rance, M.; Loria, J.P.; Palmer, A.G., III. Sensitivity improvement of transverse relaxation-optimized spectroscopy. J. Magn. Reson. 1999, 136, 92-101. [CrossRef] [PubMed]

37. Ishima, R. Recent developments in ${ }^{15} \mathrm{~N}$ NMR relaxation studies that probe protein backbone dynamics. Top. Curr. Chem. 2012, 326, 99-122. [CrossRef] [PubMed]

38. Delaglio, F.; Grzesiek, S.; Vuister, G.W.; Zhu, G.; Pfeifer, J.; Bax, A. NMRPipe: A multidimensional spectral processing system based on UNIX pipes. J. Biomol. NMR 1995, 6, 277-293. [CrossRef] [PubMed]

(C) 2018 by the authors. Licensee MDPI, Basel, Switzerland. This article is an open access article distributed under the terms and conditions of the Creative Commons Attribution (CC BY) license (http://creativecommons.org/licenses/by/4.0/). 